than normaltherapy group (562 m vs $513 \mathrm{~m}, \mathrm{p}<0.05$ ) at 3 months after operate. Still after 6 months, the left ventricular ejection fraction were more higher in Buchang group than normaltherapy group $(65.3 \%$ vs $60.8 \%, p<0.05)$. 6 miniters walk distence test were more higher in Buchang group than normaltherapy group (541 m vs $502 \mathrm{~m}$, $p<0.05)$. Moreover after 3 months, the level of sCD40L were lower in Buchang group than normaltherapy group $(2.23 \mathrm{ng} / \mathrm{ml}$ vs $2.62 \mathrm{ng} / \mathrm{ml}$, $\mathrm{p}<0.01$ ), the numbers of EPCs were more in Buchang group than normaltherapy group $(37.1 \pm 5.8$ vs $32.4 \pm 4.6, \mathrm{p}<0.05)$.

Conclusion These study suggested that Danhong injection combined naoxintong pills could decreased SCD40L level, increased EPCs level, improved cardia function at ACS patients with PCI therapy, It was worthy for deeply research.

\section{e0317 COMPARATIVE EFFECTIVENESS OF RENIN ANGIOTENSIN SYSTEM BLOCKADES PLUS CCBS OR DIURETICS FOR ESSENTIAL HYPERTENSION A SYSTEMATIC REVIEW}

doi:10.1136/hrt.2010.208967.317

Ma Ruixin, Yu Jing, Xu Dian, Yang Longquan, Liu Peijun, Bai Feng. The Second Hospital of Lanzhou University

Backround The relative effectiveness of two combination therapyRenin Angiotensin System (RAS) blockades/calcium channel blockers (CCBs) versus RAS blockades/diuretics for lowering blood pressure is unknown. This systematic review is to compare the benefits and harms of RAS blockades plus CCBs versus RAS blockades plus diuretics for treating essential hypertension in adults. Methods We retrieved MEDLINE, the Cochrane Central Register of Controlled Trials, EMBASE and SCI using computer to identify relevant randomised controlled trials in English that directly compared the effect of RAS blockades plus CCBs with that of RAS blockades plus diuretics in adult patients with essential hypertension, reported an outcome of mean difference of BP reduction or interest, lasted at least 4 weeks, and included at least 20 patients. A standardised protocol with predefined criteria was used to extract data on study design, interventions, population characteristics, and outcomes; We evaluated study quality and applicability; and assessed the strength of the evidence for key outcomes.

Results Five clinical studies were eventually included. We found no significant difference between RAS blockades/CCBs with RAS blockades/diuretics in reduction of blood pressure. However, RAS blockade/ $\mathrm{CCBs}$ associated with significant stronger DBP response rate. No differential effects were observed for the incidence of adverse events. Conclusion Available evidence shows that RAS blockade/CCBs and blockade/diuretics have similar effects on blood pressure control. High strength of evidence is needed. Data regarding is patient subgroups were missing.

\section{e0318 PITAVASTATIN CALCIUM IMPROVES CAROTID ARTERIOSCLEROSIS IN PATIENTS WITH HYPERCHOLESTEROLAEMIA}

doi:10.1136/hrt.2010.208967.318

Yan Hui-Min, Wang Jia, Wang Hua, Wang Zhi-Hao, Zhang Wei, Zhang Yun, Zhong Ming. Key Laboratory of Cardiovascular Remodelling and Function Research, Chinese Ministry of Education and Chinese Ministry of Public Health, Department of Cardiology, Qilu Hospital of Shandong University, Jinan, China

Objective Hence, our aims were to elucidate the changes of the carotid arterial structure and functions in patients with the HC. The purpose of the present study was to investigate the effect of pitavastatin calcium on artherosclerosis of carotid artery in patients with hypercholesteremia (HC).

Methods A total of 40 patients with $\mathrm{HC}$ were administered pitavastatin calcium $1 \mathrm{mg}$ or $2 \mathrm{mg}$ daily for 8 weeks and thirty healthy subjects were chosen as controls. Carotid atherosclerosis was evaluated by high-resolution B-mode ultrasonography $(5-\mathrm{MHz}$ linear array transducer; Sequia 512, Siemens). The right and left common carotid arteries (CCA) were assessed in the antero-oblique direction. For each study the following parameters were assessed and calculated using the following formulae, respectively: (1) IMT, (2): Vs, Vd, Vm, (3):Ep [Pressure-strain elastic modulus, $\mathrm{E} p=\{(\mathrm{SBP}-\mathrm{DBP}) /(\mathrm{Dd}-\mathrm{Ds}) \times$ $\mathrm{Dd}\}, \mathrm{Ep}^{*} \mathrm{Ep}^{*}=\mathrm{Ep} / \mathrm{DBP}(4) \beta:\{\beta=\mathrm{In}(\mathrm{SBP} / \mathrm{DBP}) /[(\mathrm{Dd}-\mathrm{Ds}) / \mathrm{Dd}]\},(5):$ AC $[$ Arterial compliance, $\mathrm{AC}=\pi(\mathrm{Ds} \times \mathrm{Ds}-\mathrm{Dd} \times \mathrm{Dd}) / 4 \times($ SBP-DBP $)]$, (6) RI:[RI=(Vs-Vd)/Vs], (7)PI:[PI=(Vs-Vd)/Vm], (8)plaque index.

RESULTS (1) As compared with healthy control group, IMT, $\beta$ in the HC group was significantly higher $(p<0.001)$, while Vs, RI, AC in the HC group was markedly lower $(p<0.01)$. (2) In HC patients, $\beta$ level was significantly reduced $(p<0.001)$, and, RI, AC were improved significantly ( $<<0.05, p<0.001$ respectively) 8 weeks after treatment as compared with those before treatment, however, no significant difference was noted in IMT.

Conclusions Our findings demonstrated that (1) Significant alterations to carotid arteries structure and functions were found in the HC patients, including thickened IMT, distended vessel diameter, reduced elasticity and augmented stiffness. (2) Carotid arterial functions were markedly ameliorated after treatment with pitavastatin calcium for 8 weeks.

\section{e0319 PREDICTING 30-DAY MORTALITY AMONG PATIENTS HOSPITALISED FOR DECOMPENSATED HEART FAILURE}

doi:10.1136/hrt.2010.208967.319

${ }^{1}$ Zhao Yusheng, ${ }^{1}$ Wu Xingli, ${ }^{1}$ Xue Qiao, ${ }^{1}$ Gao Lei, ${ }^{2}$ Lin Haili, ${ }^{1}$ Wang Shiwen. ${ }^{1}$ Institute of Geriatric Cardiology, Chinese Pla General Hospital; ${ }^{2}$ Chinese Pla General Hospital

Objective We investigated clinical correlates of in-hospital mortality and comorbidity of patients demonstrating heart failure progression in a large population.

Methods We included 6,949 patients with demonstrating heart failure who were hospitalised from the period of January 1, 1993, to December 31, 2007, at Chinese PLA General Hospital in Beijing. Hospital mortality and comorbidities were examined for the patients primarily admitted for decompensated HF.

Results The 30 -day in-hospital mortality was $5.4 \%$ in patients. Cox regression multivariate analysis showed that a history of cor pulmonale, stroke, renal failure, cirrhosis of liver-myocardial infarction, pneumonia, gastrointestinal bleeding and multiple organ dysfunction syndromes and age older than 65 years were the only independent predictors of in-hospital mortality. Using the regression coefficient as a benchmark, we calculated a convenient score. Nearly $23 \%$ of the patients with the score $>6$ died compared with only $1.2 \%$ of the patients with the score of 0 .

Conclusion Medical comorbidity at admission or age older than 65 years is an independent risk factor for 30-day mortality in patients with heart failure. The study illustrates that medical comorbidities at admission have proved to be a major prognostic marker for immediate poor outcome in the patients with heart failure. The score may help to identify patients who are more likely to have a risk of in-hospital mortality within 30-days.

\section{e0320 IMPACT OF PLAQUE MORPHOLOGY ON INTIMAL HYPERPLASIA AFTER STENTING ASSESSED BY OPTICAL COHERENCE TOMOGRAPHY}

doi:10.1136/hrt.2010.208967.320

Jin Qinhua, Chen Yundai, Akasaka, Mizukoshi, Kitabata, Liu Changfu, Tian Feng, Chen Lian, Sun Zhijun, Liu Hongbin, Wang Zhifeng, Guo Jun, Wang Jinda. Chinese Pla General Hospital

Objective The objective of this study was to evaluate whether the plaque morphology can affect the in-stent neointimal hyperplasia. 
Methods 74 patients (93 stents) with OCT post-stent implantation were included in the study. Cross-sectional OCT images were analysed at $1-\mathrm{mm}$ intervals (every 15 frames), and 302 crosssectional images with lipid or calcific lesions under the stent struts were selected. The struts in these sections were divided into four groups according to the plaque conditions under the struts: group 1 -struts on the normal vessel, group 2-on the fibrotic lesion, group 3-on the calcific lesion, group 4-on the lipid rich lesion. The neointimal hyperplasia thickness on the stent struts were measured by OCT.

Results The plaque conditions under 806 struts could be detected clearly by OCT. Among them, 157 struts were on the normal vascular wall (the intimal thickness were less than $250 \mu \mathrm{m}$ by OCT), 344 struts were on the fibrotic lesions, 145 struts on calcific lesions and 160 struts on lipid lesions. The neointimal thickness were $0.132 \pm 0.081 \mu \mathrm{m}, \quad 0.148 \pm 0.091 \mu \mathrm{m}, \quad 0.150 \pm 0.105 \mu \mathrm{m}$ and $0.166 \pm 0.088 \mu \mathrm{m}$ respectively in group $1-4$. The $\mathrm{p}$ value was 0.011 . Conclusions The plaque type has impact on the in-stent neointimal hyperplasia. The underlying lipid or calcific components in plaque may promote neointimal hyperplasia after stent implantation.

\section{E0321 THE CORRELATION OF CARDIAC TROPONIN I AND DECOMPENSATED HEART FAILURE}

doi:10.1136/hrt.2010.208967.321

Zhaoxiufen Sunxiaofei, Zhaoxiufen Sunxiaofei. The First People's Hospital, Shan Dong Ji Ning

Objective Correlation of Cardiac Troponin I and Decompensated heart failure. Small studies report that $\mathrm{cTnI}$ is elevated in severe heart failure (HF) and may predict adverse outcomes.

Methods Serial blood samples were instantly collected from 336 patients who presented Decompensated heart failure (NYHA class III-IV) at Admission. Patients with acute myocardial infarction or myocarditis were excluded from analysis. Measurement of cTnI and Brain natriuretic peptide (BNP), echocardiography was done after Disease condition Improved. cTnI was detectable (cTnI $\geq 0.40 \mathrm{ng} /$ $\mathrm{ml}$ ) in serum of 161 patients (47.9\%). Patients with detectable cTnI levels had significantly higher B-type natriuretic peptide (BNP) levels $(p<0.001)$. A significant correlation was found between detectable cTnI and progressive decline in ejection fraction over time. Furthermore, detectable cTnI was associated with increased mortality risk (RR, 2.57; 95\% CI, 1.43 to 3.78). After adjustment of other factors associated with adverse prognosis including age, sex, ejection fraction, and coronary artery disease, cTnI remained a significant predictor of death. cTnI used in conjunction with BNP further improved prognostic value.

Conclusions cTnI is associated with elevated BNP levels and progressive left ventricular dysfunction in patients with severe HF. cTnI may be a novel, useful tool in identifying patients with HF who are at increased risk for progressive ventricular dysfunction and death

\section{e0322 TO INVESTIGATE INAPPROPRIATE DISCHARGE OF}

doi:10.1136/hrt.2010.208967.322

Xinxia Zhang, Xuesong Hu, Xiangguang Xu, Weihua Fang, Changlong Pen. Department of Cardiology, Shenzhen Futian Hospital

Objective In order to avoid and decrease inappropriate discharge caused by implantable cardioverter defibrillators (ICD), we analysed the cause of inappropriate discharge.

Methods Clinical follow-up data of inappropriate discharges by ICD in 6 patients were studied. All the 6 patients were male, 2 were dual chamber ICD, while 7 were single chamber ICD. The patients were followed-up from 0.5 to 9 (averaged 4.5) years.

Results During follow-up, all the patients survived. Arrhythmias were detected 358 times, ICD treatment was performed 71 times, inappropriate discharges occurred 14 times. 2 patients who implanted ICD earlier experienced all the 14 inappropriate discharges. The causes of inappropriate discharges include oversensing sinus tachycardia (3 times), electromagnetic interference (2 times), lead insulation breaks (9 times). They accounted for $21.4 \%, 14.2 \%, 64.2 \%$ of total inappropriate discharges, respectively. Conclusion Inappropriate discharge of ICD should be taken seriously consideration.

\section{e0323 ANALYSIS OF ADVERSE EFFECTS FROM STATINS-BASED MULTI-DRUG MEDICATION}

doi:10.1136/hrt.2010.208967.323

Zhao Mei, Zhao Zhengdang, Shi Jin, Chen Yanli, Li Hong, Zou Deling, Ma Shumei, Li Xiaodong. Deptartment of Cardiology, Shengjing Hospital of China Medical University

Objective To investigate risk factors and possible mechanisms of adverse effects from statins-based multi-drug medication.

Methods The medical materials from eleven patients who suffered from adverse effects of statins-based multi-drug medication were collected and analysed.

Results 7 patients who took simvastatin $40 \mathrm{mg} /$ day for monotherapy for four weeks produced normal laboratory tests results. Four patients who took a combination of ezetimibe/simvastatin 10/ $40 \mathrm{mg} /$ day for 4 weeks, 4 patients who took a combination of simvastatin $40 \mathrm{mg} /$ day plus niacin for 8 weeks and 3 patients who took a combination of ezetimibe/simvastatin $10 / 40 \mathrm{mg} /$ day plus niacin for eight weeks or less showed hepatic dysfunction (Alt $>1.5 \mathrm{ULN})$ and/or amyasthenia (CK $>1.5 \mathrm{ULN})$ and finally discontinued medication.

Conclusion For females, age, polypharmacy and drug interactions are very important risk factors for statins-based multi-drug medication. One of the possible mechanisms is that other interacting drugs competitively inhibit the common pathway by which the drugs are metabolised, resulting in increased statins exposure, subsequent hepatotoxicity and skeletal muscle toxicity.

\section{e0324 PLATELET ACTIVATION DISTRIBUTION IN CHINESE HIGH RISK PATIENTS WITH ACUTE CORONARY SYNDROME}

doi:10.1136/hrt.2010.208967.324

${ }^{1}$ Ren Yihong, 'Chen Yundai, ${ }^{1}$ Zhao Ming, ${ }^{2}$ Chen Jinsong, ${ }^{1}$ Chen Lian, ${ }^{1}$ Liu Hongbin, ${ }^{1}$ Wang Yu, ${ }^{1}$ Snu Zhijun. ${ }^{1}$ PLA General Hospital/Cardiovascular Department; ${ }^{2}$ PLA General Hospital, Clinical Laboratory

Background All cases of ACS treated with the same doses antiplatelet remedy without considering their results from it is not an optimal remedy obviously. TEG-Mapping assay can offer us a possibility to detect the activity of platelet in general clinical therapy process.

Objectives To evaluate the antiplatelet therapy results immediately post- PCI in high risk ACS patients.

Methods All ACS patients $(n=310)$ with elective PCI took aspirin loading dose $300 \mathrm{mg}$ before PCI and followed by $100 \mathrm{mg} /$ day and clopidogrel loading dose $600 \mathrm{mg}$ before PCI and followed by $75 \mathrm{mg} /$ day therapy. Potential and residual platelet activity in ADP and AA pathway were detected respectively after $24-48$ h from PCI with modified TEG-mapping assay. 